\title{
IKB-kinase- $\varepsilon$ in the tumor microenvironment is essential for the progression of gastric cancer
}

\author{
Biao Geng ${ }^{1, *}$, Chen Zhang ${ }^{1, *}$, Chao Wang ${ }^{2}$, Ying Che ${ }^{3}$, Xianmin Mu ${ }^{1}$, Jinshun Pan ${ }^{1}$, \\ Che $\mathrm{Xu}^{1}$, Shi Hu${ }^{1}$, Jing Yang ${ }^{1}$, Ting Zhao ${ }^{1}$, Yue Xu ${ }^{1}$, Yuanfang Lv ${ }^{1}$, Hao Wen ${ }^{2}$, Zheng \\ Liu ${ }^{3}$ and Qiang You ${ }^{1,3,4,5}$ \\ ${ }^{1}$ Department of Biotherapy, Second Affiliated Hospital, Nanjing Medical University, Nanjing, Jiangsu 210011, China \\ ${ }^{2}$ Department of Surgery, Second Affiliated Hospital, Nanjing Medical University, Nanjing, Jiangsu 210011, China \\ ${ }^{3}$ Medical Center for Digestive Diseases, Second Affiliated Hospital, Nanjing Medical University, Nanjing, Jiangsu 210011, \\ China \\ ${ }^{4}$ Key Laboratory for Aging \& Disease, Second Affiliated Hospital, Nanjing Medical University, Nanjing, Jiangsu 210011, China \\ ${ }^{5}$ Department of Immunology, Nanjing Medical University, Nanjing, Jiangsu 211166, China \\ *These authors have contributed equally to this work \\ Correspondence to: Qiang You, email: Qiang.You@live.com
}

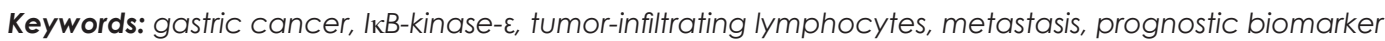

Received: June 14,2017 Accepted: July 30,2017 Published: September 08, 2017

Copyright: Geng et al. This is an open-access article distributed under the terms of the Creative Commons Attribution License 3.0 (CC BY 3.0), which permits unrestricted use, distribution, and reproduction in any medium, provided the original author and source are credited.

\section{ABSTRACT}

The tumor microenvironment is critical for tumor growth and metastasis, but the underlying molecular mechanisms are poorly understood. Recent studies have shown that IKB-kinase- $\varepsilon$ (IKK $\varepsilon$ ) is involved in the proliferation and migration of certain cancers. However, the functional role of IKK $\varepsilon$ in the progression of gastric cancer (GC) remains unknown. In this study, we found that high levels of IKKE expression in GC tumors were correlated with more advanced disease and poor overall survival of patients. Silencing of IKKE effectively suppressed the migratory and invasive capabilities of human GC cells in vitro and tumorigenicity and metastasis in vivo. Further analysis revealed that IKK $\varepsilon$ was also highly expressed in tumor-infiltrating lymphocytes. Moreover, it was involved in tumor-infiltrating T-cell-mediated invasion and metastasis. Knockdown of IKKE elevated T-cell antitumor immunity. These findings suggest that IKKE may be a novel prognostic marker and a potential therapeutic target in human GCs.

\section{INTRODUCTION}

Gastric cancer (GC) is the leading cause of cancerrelated death in China [1]. At the time of initial diagnosis, a large majority of patients have already reached an advanced stage in which tumor cell spreading has occurred, and approximately $50 \%$ of patients with $\mathrm{GC}$ will die from the development of distant metastases [2, 3]. To improve the early diagnosis of GC and targeted therapy, an in-depth understanding of the molecular underpinnings of the disease is required [4]. It is of clinical importance to identify genes that contribute to GC development and present predictive values for diagnosis or prognosis.

The nuclear factor (NF)- $\mathrm{kB}$ pathway is a pivotal regulator of several important physiological functions, including the inflammatory immune response, proliferation, cell survival and cell invasion $[5,6]$. These activities are well-described hallmarks of cancer, and NF$\kappa \mathrm{B}$ activation has been observed in a wide range of tumors, leading some to suggest that NF- $\mathrm{KB}$ serves as a bridge between inflammation and cancer [7-10]. I- $\mathrm{KB}$ kinases (IKKs), IKK $\varepsilon$ and TBK1, are key regulators of NF- $\mathrm{KB}$ 
signaling [5]. The IKK-related kinases have recently been recognized as NF- $\mathrm{KB}$ effectors that contribute to tumorigenesis and thus represent a link between NF-кBmediated inflammation and cancer [11-13]. Studies in animal models have shown that NF- $\mathrm{KB}$ is often essential for cancer initiation and progression $[11,14]$. Previous studies have suggested that IKKE is overexpressed in tumor tissue from a variety of human tumor types, such as breast cancer, non-small cell lung cancer, and pancreatic cancer, where it has been proposed as biomarker and potential therapeutic target [15-18]. However, a comprehensive understanding of how IKK $\varepsilon$ promotes tumorigenicity is lacking. IKK $\varepsilon$ is also expressed in immune cells, and may play a special role in the immune response $[11,19,20]$. Furthermore, IKK $\varepsilon$ is a crucial negative regulator of T-cell activation and a potential target for immunotherapy [21]. However, the function of IKK $\varepsilon$ remains obscure in tumor-infiltrating regulatory T-cells, despite its abundant expression. Therefore, we set out to more fully understand how IKK $\varepsilon$ controls tumorinfiltrating lymphocyte crosstalk in GC metastasis.

In the present study, we report that, in addition to the fact that IKK $\varepsilon$ is aberrantly overexpressed in GC and could coordinately serve as a promising predictive biomarker for prognosis in patients with GC, knockdown of IKK $\varepsilon$ elevates T-cell antitumor immunity and reduces tumor development. Furthermore, the functional role of IKK $\varepsilon$ in GC provides a mechanistic basis for its potential as a therapeutic target.

\section{RESULTS}

\section{IKKe is upregulated in GC tissues and is correlated with GC progression}

To examine the significance of IKKe in GC development, we first measured IKK $\varepsilon$ expression in 2 GC samples using IHC. IKK $\varepsilon$ was significantly upregulated in $\mathrm{GC}$ tissues compared with adjacent non-cancerous gastric tissues (Figure 1A). Furthermore, compared with lymphocytes from adjacent non-tumor tissues, there was an obvious increase of IKK $\varepsilon$ expression in tumor-infiltrating lymphocytes from $\mathrm{GC}$ tissues (Figure 1B). To further investigate the association of IKK $\varepsilon$ and GC progression, tissue microarray-based IHC study of IKK $\varepsilon$ in $100 \mathrm{GC}$ tissues with clinicopathological features and complete follow-up data was performed. As shown in Table 1, high expression of IKK $\varepsilon$ was found to be significantly associated with poor differentiation $(P=0.021)$, depth of invasion $(P=0.034)$, lymph node metastasis $(P<0.001)$, distant metastasis $(P=0.006)$, and tumor-node-metastasis (TNM) stage $(P=0.005)$.

\section{Tumor}

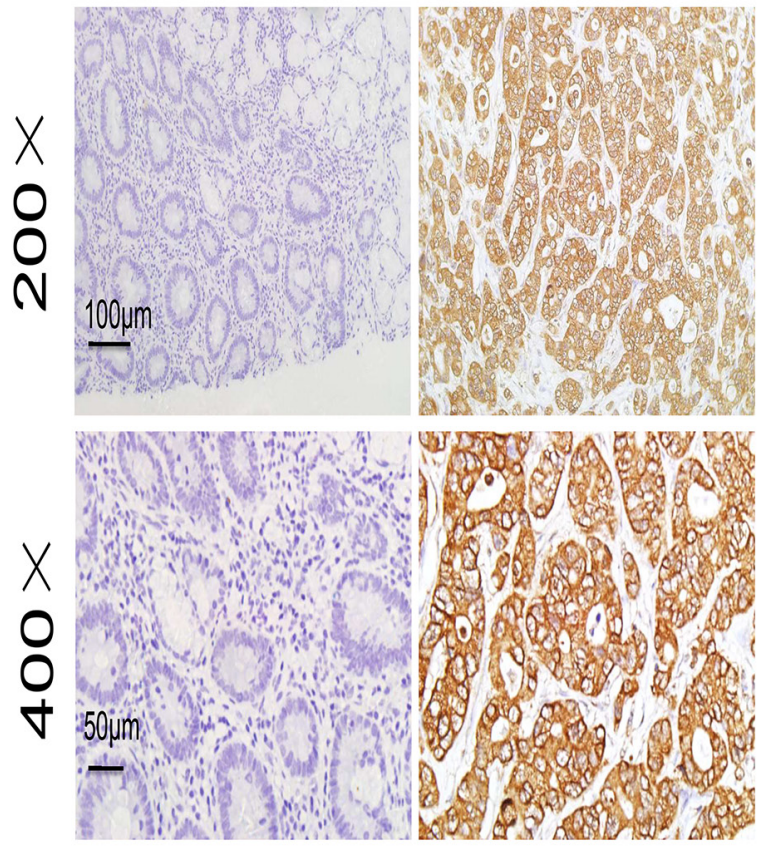

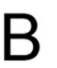

Normal lymphocytes
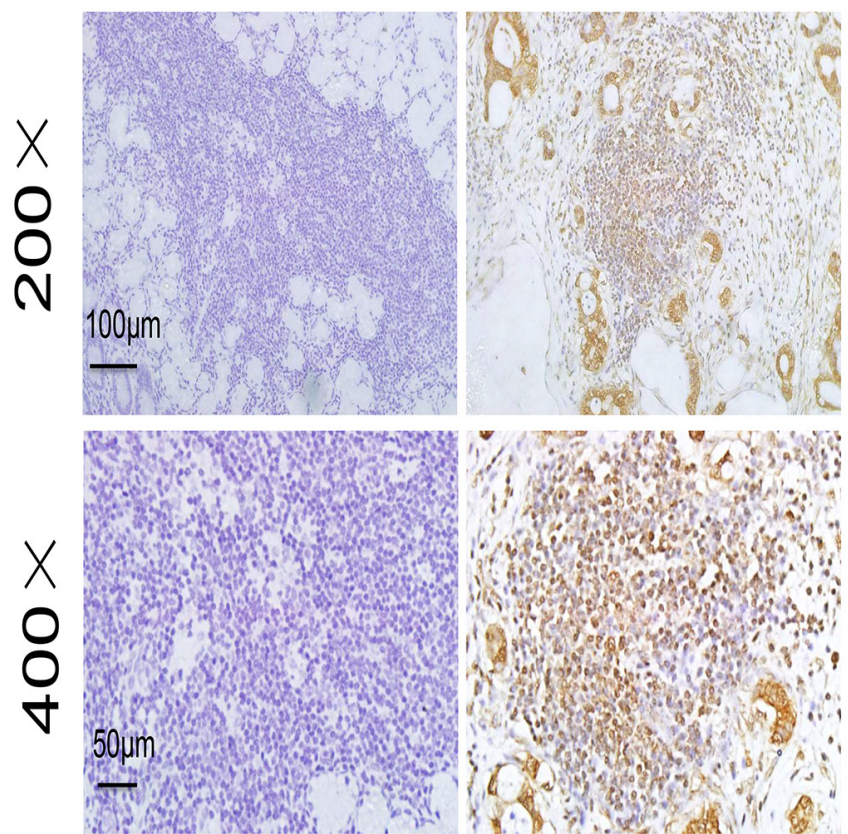

Tumor-infiltrating lymphocytes

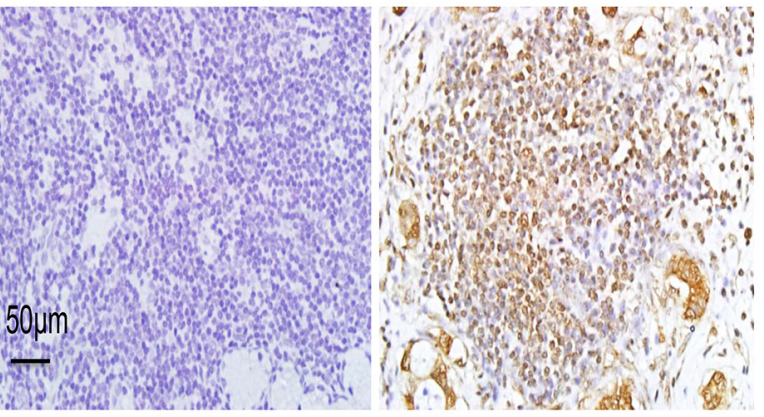

Figure 1: IKKE expression in the tumor microenvironment. (A) Representative images of IKK $\varepsilon$ staining in adjacent non-tumor tissues and GC tissues. (B) Representative images of IKK $\varepsilon$ staining in lymphocytes from adjacent non-tumor tissues and tumor-infiltrating lymphocytes from GC tissues. 
Table 1: Association between IKKe expression and clinicopathological factors in gastric cancer

\begin{tabular}{|c|c|c|c|c|}
\hline \multirow[t]{2}{*}{ Variable } & \multirow[t]{2}{*}{$\mathbf{n}$} & \multicolumn{2}{|c|}{ IKKe expression } & \multirow[t]{2}{*}{$P$ value } \\
\hline & & Low & High & \\
\hline Age & & & & 0.610 \\
\hline$\leq 60$ & 43 & 18 & 25 & \\
\hline$>60$ & 57 & 21 & 36 & \\
\hline Gender & & & & 0.674 \\
\hline Male & 72 & 29 & 43 & \\
\hline Female & 28 & 10 & 18 & \\
\hline Differentiation & & & & 0.021 \\
\hline Well & 12 & 9 & 3 & \\
\hline Moderate & 65 & 21 & 44 & \\
\hline Poor & 23 & 9 & 14 & \\
\hline Depth of invasion ( $T$ ) & & & & 0.034 \\
\hline $\mathrm{T} 1-\mathrm{T} 2$ & 36 & 19 & 17 & \\
\hline $\mathrm{T} 3-\mathrm{T} 4$ & 64 & 20 & 44 & \\
\hline $\begin{array}{l}\text { Lymph node } \\
\text { metastasis }\end{array}$ & & & & 0.000 \\
\hline N0-N1 & 33 & 28 & 15 & \\
\hline N2-N3 & 67 & 11 & 46 & \\
\hline $\begin{array}{l}\text { Distant metastasis } \\
\text { (M) }\end{array}$ & & & & 0.006 \\
\hline Negative (M0) & 78 & 36 & 42 & \\
\hline Positive (M1) & 22 & 3 & 19 & \\
\hline Tumor stage & & & & 0.005 \\
\hline I-II & 37 & 21 & 16 & \\
\hline III-IV & 63 & 18 & 45 & \\
\hline
\end{tabular}

To estimate the clinical prognostic significance of IKK $\varepsilon$ expression that might influence the overall survival of patients enrolled in this study, Kaplan-Meier survival analysis was performed in the cohort. As shown in Figure 2 , patients with higher expression of IKK $\varepsilon$ in tumor tissues were prone to lower overall survival (OS). Low expression of IKKE has a survival benefit compared with high expression (Figure 2A, $P<0.001$ ). Kaplan-Meier analysis was also applied to compare overall survival according to IKK $\varepsilon$ expression in different clinicopathological factors. Significant differences were found in N2-N3, T3-T4, and III-IV stage tumors according to IKK $\varepsilon$ expression (Figure $2 \mathrm{~B}, P=0.008$, Figure $2 \mathrm{C}, P=0.015$, Figure $2 \mathrm{D}, P=$ $0.019)$. Moreover, Cox regression analysis also indicated that high IKK $\varepsilon$ expression was an independent prognostic factor for poor survival in GC patients (Table 2). Together, these results suggest that IKKE overexpression was significantly associated with poor prognosis of GCs.

\section{IKK $\varepsilon$ regulates cell proliferation, migration, and} invasion

We first examined the expression level of IKK $\varepsilon$ in a panel of human GC cells. The results indicated that the protein expression of IKK $\varepsilon$ was higher in SGC7901and MGC803 cells (Figure 3A). To identify the potential function of IKKe in GC cell growth and metastasis, SGC7901 and MGC-803 cells with stably knockeddown IKK $\varepsilon$ were created. Changes in IKK $\varepsilon$ expression were confirmed using western blotting (Figure 3A). The analysis of cell proliferation by the CCK8 assay revealed that compared with control cells, IKK $\varepsilon$ knockdown cells had lower proliferation (Figure 3B) and colony-formation rates (Figure 3C). The number of cells that invaded through the Matrigel or migrated was clearly decreased for IKK $\varepsilon$ knockdown cells in the Transwell assay compared to control cells (Figure 3D). The wound closure assay 
verified the results, which showed that wound recovery was significantly impaired by IKKe knockdown in comparison with the controls (Figure 3E). Together, these experiments identified IKK $\varepsilon$ as the critical oncoprotein that mediates GC cell proliferation and invasion.

\section{Knockdown of IKKE elevates T cell antitumor immunity and reduces tumor development}

Recent studies have demonstrated that IKKe is expressed in immune cells and may play a special role in regulating T-cell function $[11,14,21]$. As a consequence,

A

All patients

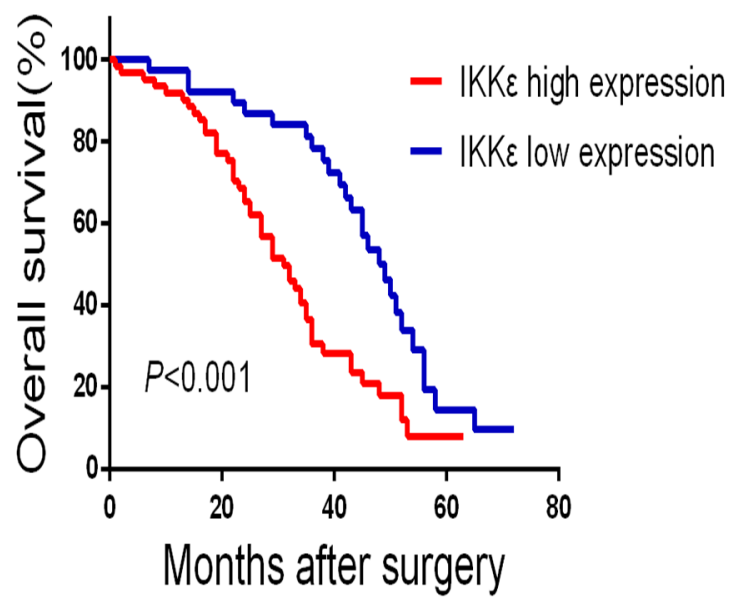

C

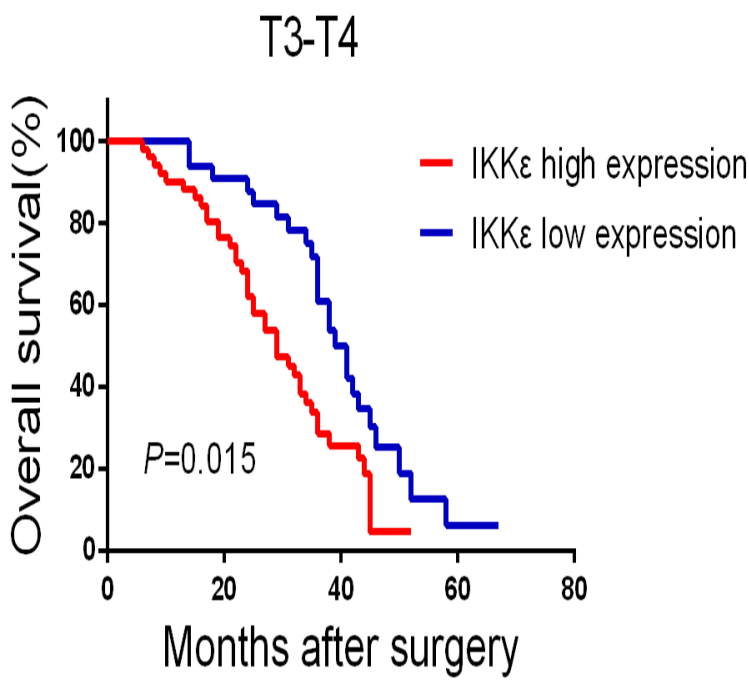

studies were undertaken to determine whether IKKE upregulation plays a role in promoting tumor metastasis. To accomplish this, we compared the B16-F10 melanoma cell-induced metastasis in WT mice and IKK $\varepsilon$ null mice. Melanoma cell administration caused impressive levels of metastasis in the lungs of WT mice, and this metastatic response was markedly decreased in IKK $\varepsilon$ null mice (Figure 4A, 4B). To investigate whether knockdown of IKK $\varepsilon$ influences T-cell function, we examined the content of $\mathrm{CD}^{+} \mathrm{T}$-cells in bronchoalveolar lavage fluid. The results revealed that $\mathrm{CD} 8^{+} \mathrm{T}$-cells in the WT mice were significantly decreased compared to mice with null

B

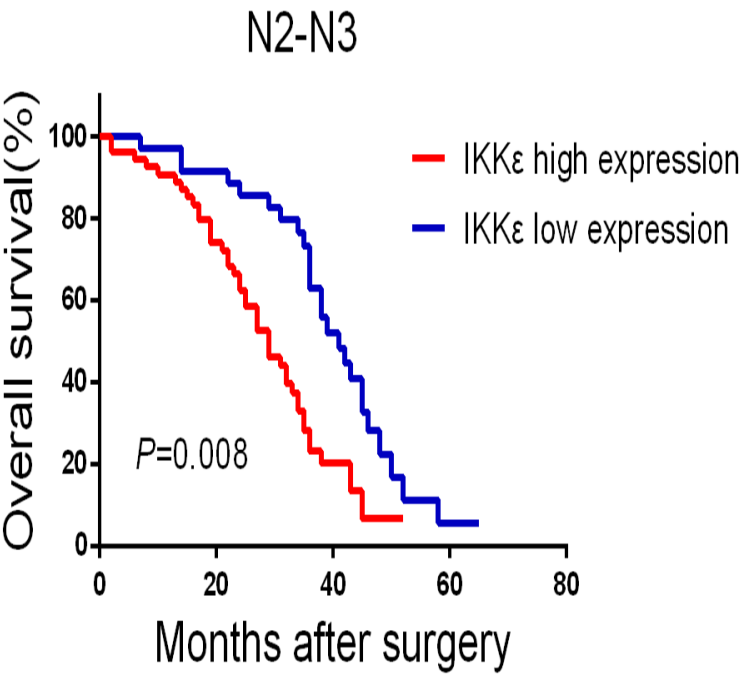

D

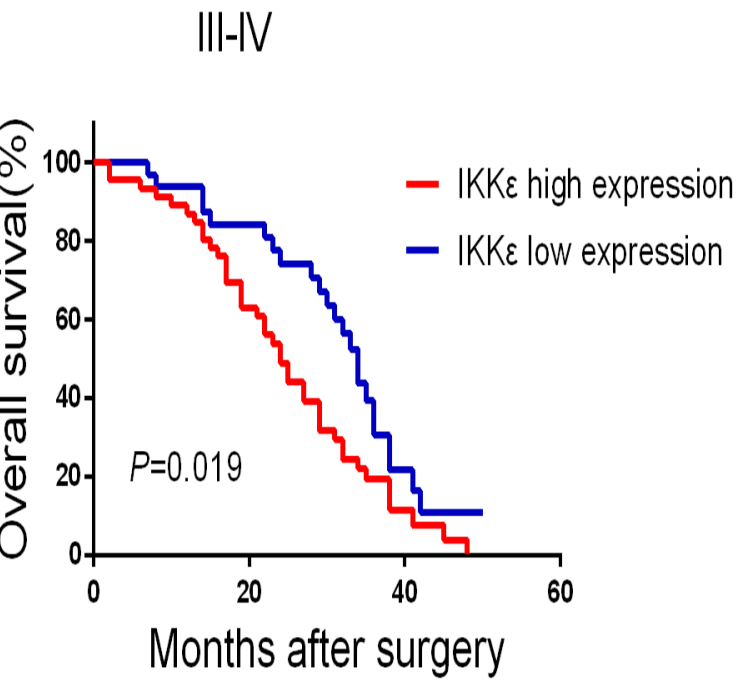

Figure 2: IKKE overexpression is associated with poor prognosis in GC patients. (A) Kaplan-Meier analysis for OS of patients with gastric cancer according to IKK $\varepsilon$ expression in all patients, patients with N2-N3 stage tumors (B) patients with T3-T4 stage tumors (C), and patients with III-IV stage tumors (D). 
Table 2: Multivariate analysis for overall survival

\begin{tabular}{lccc}
\hline Variable & HR & CI (95\%) & P value \\
\hline Depth of invasion & 1.236 & $0.924-2.256$ & 0.121 \\
Lymph node metastasis & 2.374 & $1.237-3.562$ & 0.026 \\
Distant metastasis & 4.518 & $2.331-8.318$ & 0.001 \\
Tumor stage & 5.231 & $3.169-7.573$ & 0.000 \\
Differentiation & 1.252 & $0.726-1.842$ & 0.535 \\
IKKE expression & 2.013 & $1.204-7.471$ & 0.014 \\
\hline
\end{tabular}

mutations of IKKe (Figure 4C). Additionally, the survival of the IKK $\varepsilon$ null mice was increased compared with WT controls (Figure 4D). When viewed together, these studies demonstrate that knockdown of IKK $\varepsilon$ results in elevated numbers of activated $\mathrm{CD}^{+} \mathrm{T}$-cells in the tumor microenvironment.

\section{IKKE promotes tumor growth, invasion, and metastasis in vivo}

To confirm these results of experiments in vitro, we further evaluated the functional role of IKK $\varepsilon$ expression on in vivo tumor growth and metastasis of GC cells. We first developed subcutaneous xenograft tumor models in nude mice by subcutaneous injection of MGC803 cells infected with scrambled or IKKe shRNA. As shown in Figure $5 \mathrm{~A}$ and $5 \mathrm{C}$, the size of the xenograft tumors derived from the IKKe knockdown MGC803 cells was significantly smaller than those formed by control cells. The weights of the xenograft tumors corresponded to their sizes (Figure 5D).

To investigate whether IKK $\varepsilon$ promoted tumor metastasis in vivo, we injected SGC7901 cells into the lateral tail vein of nude mice for assessment of metastasis. Histological examination validated pulmonary metastasis (Figure 5E). We found that IKK $\varepsilon$ knockdown significantly reduced the area of metastatic lesions that appeared in the lungs of the mice (Figure 5F).

Considering these data together, our experiments revealed that reduction of IKKe protein expression effectively interfered with the potential of GC cells to proliferate and metastasize in vivo.

\section{DISCUSSION}

GC metastasis is the main cause of GC-related mortality. However, its mechanism remains poorly understood. It is a complex multistep process, involving alterations in the dissemination, invasion, survival, and growth of new cancer cell colonies, which are regulated by a complex network of intra- and inter-cellular signal transduction cascades $[23,24]$. In this study, we have disclosed that IKK $\varepsilon$ upregulation is significantly associated with a more aggressive tumor phenotype. Cox regression analysis also indicated that high IKKE expression is an independent prognostic factor for poor survival in GC patients. We also revealed the underlying mechanism for such an association by demonstrating IKK $\varepsilon$ as a crucial negative regulator of $\mathrm{T}$-cell activation.

IKKع's play essential roles as regulators of proper immune function, cell survival, apoptosis, and cellular proliferation by modulating the NF- $\mathrm{B}$ pathway [25]. It is critical for activation of NF-kB complexes downstream of cytokine signaling and through oncoprotein expression [26]. Recent studies have also implicated IKKع's impact on cell proliferation and transformation, and it is thereby also classified as an oncogene [5]. It has been suggested that IKKe is aberrantly expressed in approximately $30 \%$ of breast carcinomas, in which it induces survival signaling associated with $\mathrm{NF}-\kappa \mathrm{B}$ pathway activation [18]. Furthermore, IKK $\mathrm{K}$-associated cytokine signaling promotes tumorigenicity of immune-driven triplenegative breast cancers (TNBCs) [15]. Indeed, IKKع's have emerged as a critical modulator of cancerous traits, yet little is known about its significance and expression in GC. Here, we have demonstrated that high expression of IKK $\varepsilon$ was found to be significantly associated with GC progression. Therefore, IKK $\varepsilon$ has potential for utilization as a predictive marker for GC patient outcomes. The tumor-facilitating functions of IKKE that have been described so far include the following: IKK $\varepsilon$ may contribute to enhanced NF-kB activity and tumorigenesis by directly phosphorylating NF-kB p65 or by phosphorylating Akt, which then phosphorylates and activates p65 [27]. Moreover, elevated IKK $\varepsilon$ directly phosphorylates and activates specific STAT transcription factors in different primary tumors and cell lines derived from a diversity of cancers, such as lung and breast carcinoma, which may contribute to the oncogenic activation of IKK $\varepsilon[17,18,27]$.

To further understand the biological function of IKK $\varepsilon$ in GC progression, we investigated the malignant features of IKKe in GC cell lines. Our data showed that knockdown of IKK $\varepsilon$ completely reversed the effect of IKK $\varepsilon$ in proliferation, colony formation, and migration and invasion assays. Decreased IKKE 
expression also reduced the growth and metastasis of GC xenografts in nude mice. These in vivo findings correlate well with in vitro results that IKKe functions as an inducer of GC metastasis and is correlated with clinical stage, lymph node metastasis, and prognosis in GC patients.

The tumor microenvironment, consisting of extracellular matrix (ECM), fibroblasts, vasculature, and
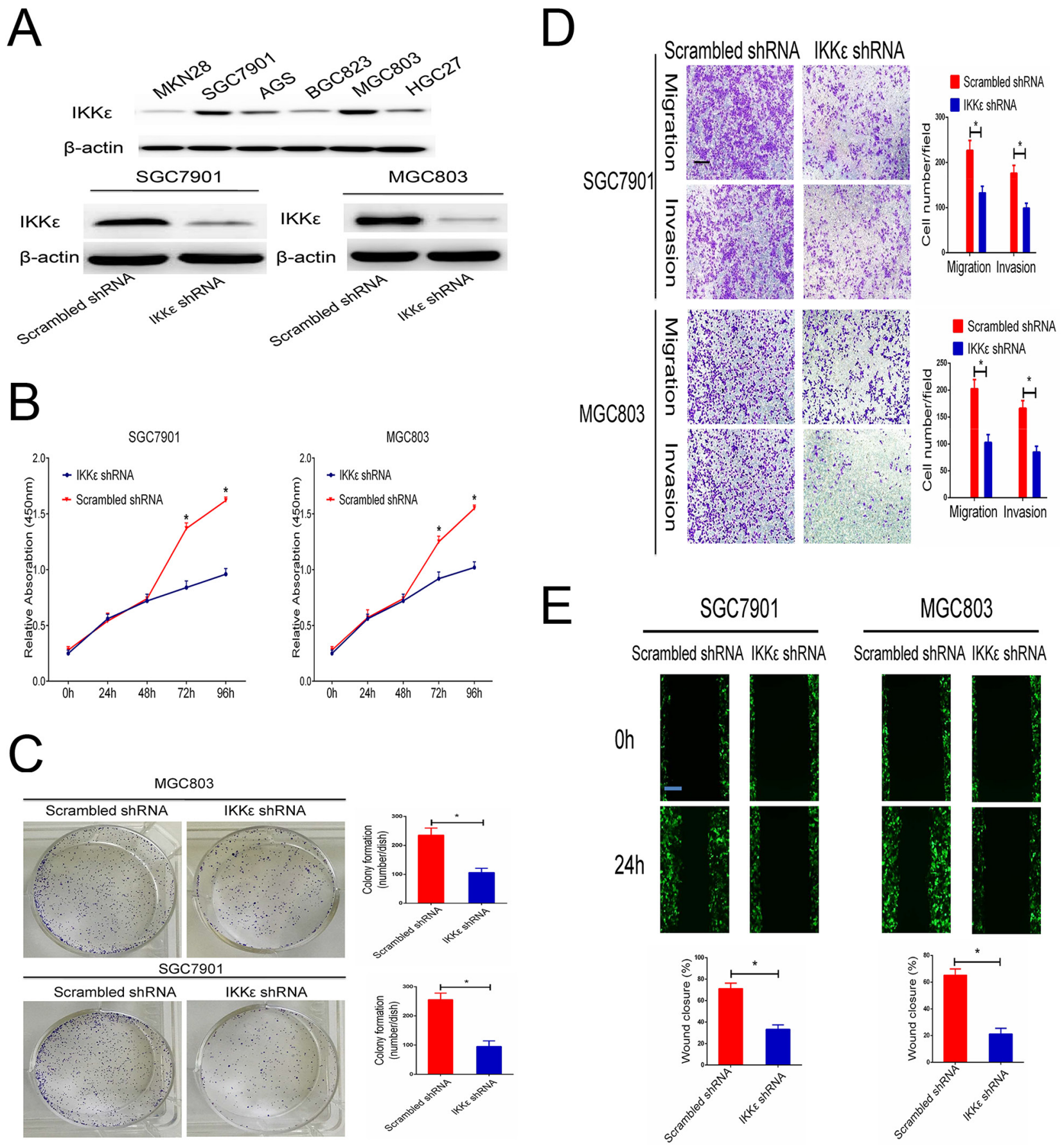

Figure 3: IKKe enhances the invasion and migration of GC cell lines. (A) The expression of IKKe was analyzed by Western blotting GC cell lines. SGC7901 and MGC803 cells were transfected with IKK $\varepsilon$ shRNA or scrambled shRNA. The efficacy of knockdown was assessed by Western blot. (B) CCK-8 assays were used to analyze the proliferation of GC cells transfected with IKK $\varepsilon$ shRNA or scrambled shRNA. (C) Plate clone formation efficiencies of cells in the presence of IKK $\varepsilon$ shRNA were compared with negative controls. (D) Transwell assay in SGC7901 and MGC803 cells stably transfected with IKK $\varepsilon$ shRNA or scrambled shRNA. Scale bars, 100 $\mu$ m. (E) Wound healing assays for SGC7901 and MGC803 cells transfected with IKK $\varepsilon$ shRNA or scrambled shRNA. Scale bars, 100 $\mu$ m. Data from 3 independent experiments were presented as mean $\pm \mathrm{SD}$. $* P<0.05$. 
A

$|K K \varepsilon-/-\quad \quad| K K \varepsilon+/+$
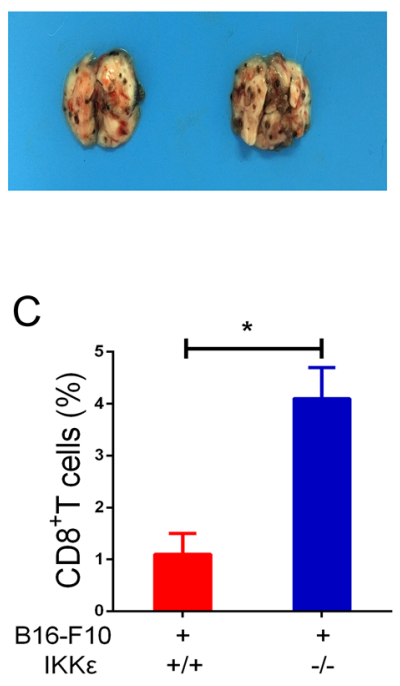

B

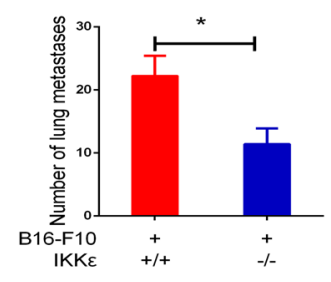

D

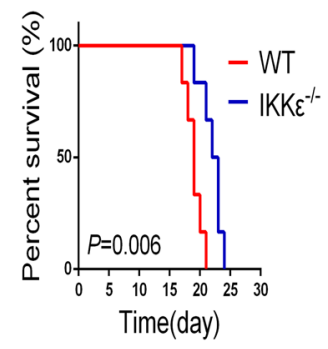

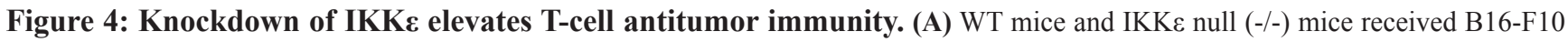
melanoma cells by tail-vein injection. Two weeks later, melanoma metastasis was visually assessed ( $\mathrm{n}=6 /$ group). (B) Quantification of pleural melanoma colonies. (C) The isolated cells from bronchoalveolar lavage fluid (BALF) were analyzed by CD8 staining. (D) Mouse survival was shown by Kaplan-Meier survival curves. ${ }^{*} P<0.05$.

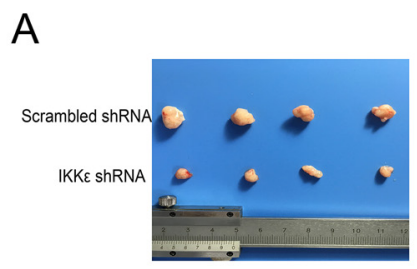

B
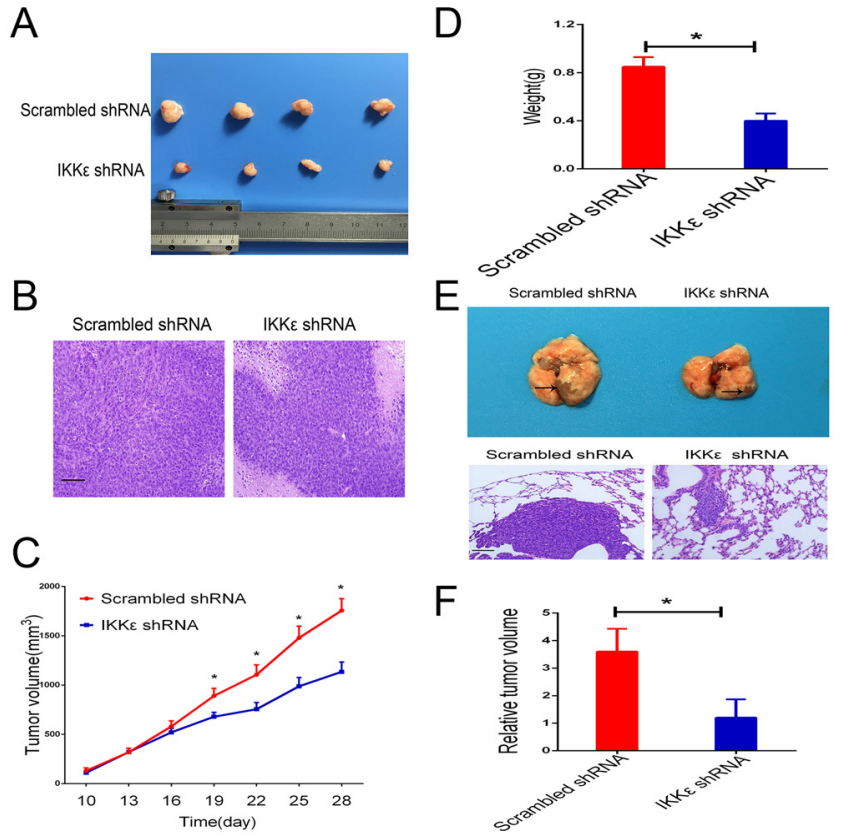

Figure 5: IKKe drives tumor growth and metastasis in vivo. (A) Representative images of tumors formed in nude mice injected subcutaneously with MGC803 cells transfected with IKK $\varepsilon$ shRNA or control shRNA (n=8/group). (B) Representative images of tumor samples stained with hematoxylin and eosin $(\mathrm{H} \& \mathrm{E})(200 \times$ magnification). Scale bars, $100 \mu \mathrm{m}$. (C) Quantification of tumor growth curves of xenograft in mice. (D) Quantification of tumor weights of xenograft in mice. (E) Representative images of metastatic tumors in lung samples ( $\mathrm{n}=5$ /group). H\&E staining of the representative metastatic lesions in nude mice $(200 \times$ magnification). Scale bars, $100 \mu \mathrm{m}$. (F) The lungs were divided into 8 parts, and 5 slides acquired from each part were used to calculate the area of metastatic lesions. The total area of invasive lesions on these slides was described as the invasive tumor volume. ${ }^{*} P<0.05$. 
tumor-infiltrating lymphocytes, is critical for tumor growth and metastasis [28-30]. Recently, an increasing amount of research has focused on the tumor microenvironment as it has become apparent that an environment rich in immune system cells, such as macrophages, T-cells, and B cells, promote tumor growth, metastasis, and relapse [24, 3133]. In this study, we observed that that loss of IKKe results in elevated numbers of activated $\mathrm{CD} 8^{+} \mathrm{T}$-cells in the tumor microenvironment. Conceivably, depletion of IKK $\varepsilon$ may elevate these signaling events to promote $\mathrm{CD}^{+} \mathrm{T}$-cell activation. Our data showed that most patients with lymph node metastasis were in the IKK $\varepsilon$-high group, whereas patients without lymph node involvement were in the IKK $\varepsilon$-low group. One possible explanation for these observations is that cancer cells might secrete a paracrine factor or factors that promote IKK $\varepsilon$ expression in tumorinfiltrating T-cells, which would inhibit T-cell immune response. Thus, the mechanism of IKK $\varepsilon$ activation, in particular, and its roles in tumor-infiltrating lymphocytes in T-cells, in general, requires investigation.

These results identify 2 major clinically relevant directions for future work. First, IKK $\varepsilon$ overexpression is significantly correlated with more advanced disease and poor survival of GC patients. Thus, IKKe could serve as a promising predictive biomarker for recurrence and prognosis in patients with GC. Second, loss of IKKe elevates T-cell antitumor immunity, which may provide a path to combined therapy that would be effective in primary tumors or established metastasis.

\section{MATERIALS AND METHODS}

\section{Cell culture}

Human GC cell lines MKN28, AGS, SGC7901, BGC823, MGC803, and HGC27 (Cell Bank of Shanghai Institute of Cell Biology, Chinese Academy of Sciences) were cultured in RPMI 1640 medium (Sigma, USA) supplemented with 10\%fetal bovine serum (FBS) (Gibco, USA), 100 units/ml penicillin, and $100 \mathrm{mg} / \mathrm{ml}$ streptomycin (Thermo Scientific). Cells were cultured at $37^{\circ} \mathrm{C}$ in a humidified $95 \%$ air, $5 \% \mathrm{CO}_{2}$ atmosphere.

\section{Immunohistochemistry (IHC)}

A GC tissue microarray containing 100 cases of GC and paired adjacent non-cancerous tissue was purchased from Shanghai Outdo Biotech (HStmA180Su08). For IHC, the target molecule was performed on tissue microarray chips using IKK $\varepsilon$ antibody (Invitrogen,\# PA5-15439). The microarray were stained with immunohistochemical streptavidin-peroxidase (SP) staining. The immunostaining index was based on the proportion of positively stained tumor cells and staining intensity. The proportion of positively stained tumor cells was graded as (no positively stained cells), $1(<10 \%), 2$
(10\%-50\%), and 3 ( $>50 \%$ of positive cells), and staining intensity was scored as 0 (no staining), 1 (light yellow), 2 (yellow brown), and 3 (brownish-yellow staining). The immunostaining index was then calculated as the staining intensity score multiplied by the proportion of positively stained tumor cells; tumors with indexes of 0 to 2 were considered immunostaining-low and those with 3 to 9 were scored immunostaining-high. Tumor stage was reassessed according to the seventh edition of the UICC/ AJCC TNM staging system.

\section{RNA interference analysis}

IKK $\varepsilon$ shRNAs (Shanghai Genechem Co., Ltd) were used to knock down IKKe according to the protocols provided by the manufacturer.

\section{Western blotting}

Immunoblots were performed following the previously described procedures. The following antibodies were used in the study [22]: IKKe (Cell Signaling Technology, \#3416) and $\beta$-actin (Cell Signaling Technology, \#4970).

\section{Cell migration and invasion assays}

In vitro invasion and migration assays were performed in 24-well Boyden chambers (Corning Incorporated, Corning, NY, USA) with or without Matrigel (BD) pre-coating. First, $600 \mathrm{ml}$ of complete medium was added to the lower chamber. Second, $200 \mathrm{ml}$ of a $2 \times 10^{5} / \mathrm{ml}$ cell suspension prepared in FBS-free Dulbecco's Modified Eagle's Medium (DMEM) was seeded into the top well of the insert, and the cell migration filter was inserted into the lower chamber; the wells were incubated for 12 to $48 \mathrm{~h}$ at $37^{\circ} \mathrm{C}$. Then, the cells on the top side of the filter were removed, the invasive and migrating cells were fixed with $500 \mathrm{ml}$ of $4 \%$ paraformaldehyde for $20 \mathrm{~min}$, and the fixed cells were stained with hematoxylin for 3 min. The invasive and migrating cells were counted and photographed under a light microscope. All experiments were conducted in triplicate.

\section{Cell proliferation}

Cell proliferation assay was performed using Cell Counting Kit -8 reagent (Whsbio, Beijing, China) according to the manufacturer's instructions.

\section{Clone formation assay}

A total of 200 cells were seeded onto wells of a 6-well culture plate. The cells were then incubated for 12 days and subsequently stained with Giemsa solution. The number of colonies containing $\geq 50$ cells in the plates was determined using the formula: plate clone formation 
efficiency $=$ (number of colonies/number of cells inoculated) $\times 100 \%$.

\section{Wound closure assay}

Six-well plates were used to seed GC cells at a density of $2 \times 10^{5}$ cells per well. Then, cells were scratched with a sterile pipette tip upon confluence. An inverted microscope was used to observe wound closure at $0 \mathrm{~h}$ and $24 \mathrm{~h}$.

\section{Assessment of melanoma lung metastasis}

After confluence of culture in ordinary DMEM, B16-F10 were delivered to IKK $\varepsilon$ null mice and wild-type (WT) mice (purchased from The Jackson Laboratory) by tail-vein injection $\left(2 \times 10^{5}\right.$ cells/mouse $)$. Lung melanoma metastases were quantified by counting the number of colonies. T-cells in bronchoalveolar lavage fluid (BALF) were analyzed by flow cytometry with antibodies against CD8.

\section{Xenograft model}

Subcutaneous xenografts were created in the flank regions of 4 -week-old nude male mice $(8$ mice per cell line). A total of $5 \times 10^{6} \mathrm{MGC}-803$ cells were implanted with or without IKK $\varepsilon$ alteration by shRNA transduction. Monitoring of tumor nodules was performed every 4 days, and tumor volumes were estimated with the following formula:Volume $=$ width $\times$ length $\times($ width + length $) / 2$. The mice were euthanized on day 28 , and tumors were removed. The animal studies were approved by the Nanjing Medical University Ethics Review Board.

\section{The metastasis of GC cells in vivo}

Stable SGC-7901 cells (transfected with IKKع shRNA or Control) were injected into nude mice via the tail vein, and the mice were sacrificed 6 weeks later for the analysis of the invasive lesions in the lungs.

\section{Statistical analyses}

The data are presented as the means \pm standard deviation (SD). Independent Student $t$-tests were used to compare the continuous variables between the 2 groups, and categorical variables were compared using the Chi-squared test. Overall survival was calculated using the KaplanMeier method and the log-rank test. Multivariate analysis was performed using the Cox proportional hazards model. Differences were considered significant if $P<0.05$.

\section{CONFLICTS OF INTEREST}

The authors declare that they have no competing interests.

\section{FUNDING}

Jiangsu Provincial Innovation Team Program Foundation (to Q.Y., D.S., H.Z.), National Natural Science Foundation of China 81471543, 81671543 (to Q.Y.), Jiangsu Provincial Natural Science Foundation BK20141488 (to Q.Y.), Jiangsu Provincial Distinguished Medical Experts Program Foundation (to Q.Y.), Preventive Medicine Foundation of Jiangsu Provincial Commission of Health and Family Planning Y2013058 (to Q.Y.), Jiangsu Provincial Six talent Peaks Program Foundation 2015-WSW-010 (to Q.Y.) and Jiangsu Provincial 333 high level talents Program Foundation CRA2016525 (to Q.Y.).

\section{REFERENCES}

1. Chen W, Zheng R, Baade PD, Zhang S, Zeng H, Bray F, Jemal A, Yu XQ, He J. Cancer statistics in China, 2015. CA Cancer J Clin. 2016; 66:115-132.

2. Glockzin G, Piso P. Current status and future directions in gastric cancer with peritoneal dissemination. Surg Oncol Clin N Am. 2012; 21:625-633.

3. Coburn N, Seevaratnam R, Paszat L, Helyer L, Law C, Swallow C, Cardosa R, Mahar A, Lourenco LG, Dixon M, Bekaii-Saab T, Chau I, Church N, et al. Optimal management of gastric cancer: results from an international RAND/UCLA expert panel. Ann Surg. 2014; 259:102-108.

4. Riquelme I, Saavedra K, Espinoza JA, Weber H, Garcia P, Nervi B, Garrido M, Corvalan AH, Roa JC, Bizama C. Molecular classification of gastric cancer: towards a pathway-driven targeted therapy. Oncotarget. 2015; 6:24750-24779. https://doi.org/10.18632/oncotarget.4990.

5. Shen RR, Hahn WC. Emerging roles for the non-canonical IKKs in cancer. Oncogene. 2011; 30:631-641.

6. Kondylis V, Kumari S, Vlantis K, Pasparakis M. The interplay of IKK, NF-kappaB and RIPK1 signaling in the regulation of cell death, tissue homeostasis and inflammation. Immunol Rev. 2017; 277:113-127.

7. Perkins ND. The diverse and complex roles of NF-kappaB subunits in cancer. Nat Rev Cancer. 2012; 12:121-132.

8. Perkins ND. Integrating cell-signalling pathways with NF-kappaB and IKK function. Nat Rev Mol Cell Biol. 2007; 8:49-62.

9. Hinz M, Scheidereit C. The IkappaB kinase complex in NF-kappaB regulation and beyond. EMBO Rep. 2014; 15:46-61.

10. Ma B, Hottiger MO. Crosstalk between Wnt/beta-catenin and NF-kappaB signaling pathway during inflammation. Front Immunol. 2016; 7:378.

11. D'Ignazio L, Bandarra D, Rocha S. NF-kappaB and HIF crosstalk in immune responses. FEBS J. 2016; 283:413-424.

12. D'Ignazio L, Rocha S. Hypoxia induced NF-kB. Cells. 2016; 5:10 
13. Gudkov AV, Komarova EA. p53 and the carcinogenicity of chronic inflammation. Cold Spring Harb Perspect Med. 2016; 6 .

14. Bradford JW, Baldwin AS. IKK/nuclear factor-kappaB and oncogenesis: roles in tumor-initiating cells and in the tumor microenvironment. Adv Cancer Res. 2014; 121:125-145.

15. Barbie TU, Alexe G, Aref AR, Li S, Zhu Z, Zhang X, Imamura Y, Thai TC, Huang Y, Bowden M, Herndon J, Cohoon TJ, Fleming T, et al. Targeting an IKBKE cytokine network impairs triple-negative breast cancer growth. J Clin Invest. 2014; 124:5411-5423.

16. Challa S, Guo JP, Ding X, Xu CX, Li Y, Kim D, Smith MA, Cress DW, Coppola D, Haura EB, Cheng JQ. IKBKE is a substrate of EGFR and a therapeutic target in non-small cell lung cancer with activating mutations of EGFR. Cancer Res. 2016; 76:4418-4429.

17. Rajurkar M, Dang K, Fernandez-Barrena MG, Liu X, Fernandez-Zapico ME, Lewis BC, Mao J. IKBKE is required during KRAS-induced pancreatic tumorigenesis. Cancer Res. 2017; 77:320-329.

18. Wei C, Cao Y, Yang X, Zheng Z, Guan K, Wang Q, Tai Y, Zhang Y, Ma S, Cao Y, Ge X, Xu C, Li J, et al. Elevated expression of TANK-binding kinase 1 enhances tamoxifen resistance in breast cancer. Proc Natl Acad Sci U S A. 2014; 111:E601-610.

19. Baldwin AS. Regulation of cell death and autophagy by IKK and NF-kappaB: critical mechanisms in immune function and cancer. Immunol Rev. 2012; 246:327-345.

20. Tuosto L. NF-kappaB family of transcription factors: biochemical players of CD28 co-stimulation. Immunol Lett. 2011; 135:1-9.

21. Zhang J, Feng H, Zhao J, Feldman ER, Chen SY, Yuan W, Huang C, Akbari O, Tibbetts SA, Feng P. IkappaB kinase epsilon is an NFATc1 kinase that inhibits $\mathrm{T}$ cell immune response. Cell Rep. 2016; 16:405-418.

22. Mu X, Zhao T, Xu C, Shi W, Geng B, Shen J, Zhang C, Pan J, Yang J, Hu S, Lv Y, Wen H, You Q. Oncometabolite succinate promotes angiogenesis by upregulating VEGF expression through GPR91-mediated STAT3 and ERK activation. Oncotarget. 2017; 8:13174-13185. https://doi. org/10.18632/oncotarget.14485.

23. Chaffer CL, Weinberg RA. A perspective on cancer cell metastasis. Science. 2011; 331:1559-1564.

24. McAllister SS, Weinberg RA. The tumour-induced systemic environment as a critical regulator of cancer progression and metastasis. Nat Cell Biol. 2014; 16:717-727.

25. Verhelst K, Verstrepen L, Carpentier I, Beyaert R. IkappaB kinase epsilon (IKKepsilon): a therapeutic target in inflammation and cancer. Biochem Pharmacol. 2013; 85:873-880.

26. Rinkenbaugh AL, Baldwin AS. The NF-kappaB pathway and cancer stem cells. Cells. 2016; 5:16.

27. Verstrepen L, Beyaert R. Receptor proximal kinases in NF-kappaB signaling as potential therapeutic targets in cancer and inflammation. Biochem Pharmacol. 2014; 92:519-529.

28. Quail DF, Joyce JA. Microenvironmental regulation of tumor progression and metastasis. Nat Med. 2013; 19:1423-1437.

29. Hanahan D, Coussens LM. Accessories to the crime: functions of cells recruited to the tumor microenvironment. Cancer Cell. 2012; 21:309-322.

30. Guo Q, Jin Z, Yuan Y, Liu R, Xu T, Wei H, Xu X, He S, Chen S, Shi Z, Hou W, Hua B. New mechanisms of tumorassociated macrophages on promoting tumor progression: recent research advances and potential targets for tumor immunotherapy. J Immunol Res. 2016; 2016:9720912.

31. Sun Y, Campisi J, Higano C, Beer TM, Porter P, Coleman I, True L, Nelson PS. Treatment-induced damage to the tumor microenvironment promotes prostate cancer therapy resistance through WNT16B. Nat Med. 2012; 18:1359-1368.

32. Kim J, Bae JS. Tumor-associated macrophages and neutrophils in tumor microenvironment. Mediators Inflamm. 2016; 2016:6058147.

33. Mantovani A, Allavena P. The interaction of anticancer therapies with tumor-associated macrophages. J Exp Med. $2015 ; 212: 435-445$. 